\title{
Coulisses
}

Revue de théâtre

12 | Printemps 1995

Varia

\section{Enseignement et recherche sur le théâtre à l'U.F.R. Lettres et Sciences Humaines de Franche-Comté}

\section{Lucile Garbagnati}

\section{(2) OpenEdition}

1 Journals

Édition électronique

URL : http://journals.openedition.org/coulisses/3469

DOI : $10.4000 /$ coulisses.3469

ISSN : 2546-9460

Éditeur

Presses universitaires de Franche-Comté

\section{Édition imprimée}

Date de publication : 1 mai 1995

Pagination : 78-85

ISSN : 1150-594X

\section{Référence électronique}

Lucile Garbagnati, «Enseignement et recherche sur le théâtre à I'U.F.R. Lettres et Sciences Humaines de Franche-Comté ", Coulisses [En ligne], 12 | Printemps 1995, mis en ligne le 15 mars 2019, consulté le 22 octobre 2019. URL : http://journals.openedition.org/coulisses/3469; DOI : 10.4000/coulisses. 3469

Ce document a été généré automatiquement le 22 octobre 2019

Coulisses 
Enseignement et recherche sur le théâtre à l'U.F.R. Lettres et Sciences

Humaines de Franche-Comté

Lucile Garbagnati

Rappel 
1 De conversations diverses, il ressortait que plusieurs d'entre nous s'intéressaient au théâtre dans le cadre de leur recherche ou de leur enseignement sans le prendre pour centre d'intérêt majeur.

Il a semblé utile de recenser ces préoccupations sous forme de questionnaire de manière à faire le point des formations et des recherches pour

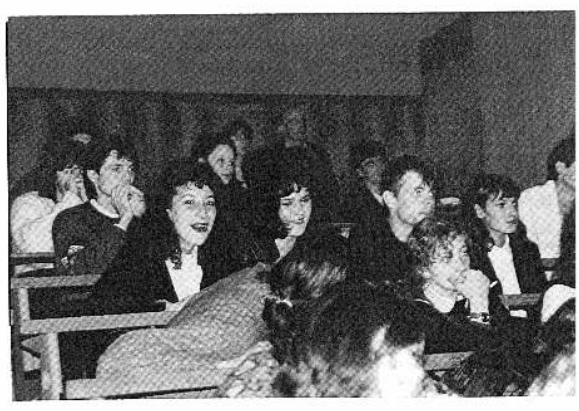
organiser éventuellement des journées d'études ou de rencontres sur des thèmes communs.

Celui-ci a été diffusé auprès de 60 collègues dont nous connaissions l'intérêt pour le théâtre et auprès des chefs de section pour qu'ils le transmettent à qui de droit. Rappelons qu'il y a 204 enseignants-chercheurs.

Après relance auprès de 40 personnes, nous avons reçu 34 réponses écrites : 4 de littérature comparée, 9 de littérature française, 12 des langues vivantes, 5 des lettres classiques, 2 de linguistique, 1 d'histoire, 1 de sociologie. Aucune réponse des géographes, des psychologues, des philosophes.

On peut avancer deux éléments d'explication à ce silence. Le théâtre n'est pas traité comme une question, mais à titre d'exemple comme il a été souligné lors de conversations privées. Les enseignants ne se sont pas sentis concernés. C'est dommage. En effet, il aurait été intéressant de savoir dans quel cadre le théâtre était utilisé comme exemple et l'intérêt qu'y trouvait l'enseignant. Autre remarque, les questionnaires ayant été envoyés au responsables de section, il n'y a pas eu diffusion. Il paraît en effet étonnant que personne ne se préoccupe du théâtre dans le cadre de l'aménagement du territoire alors qu'au moins deux salles viennent d'être construites dans ces dernières années et que la Franche-Comté compte un Centre Dramatique National et trois "scènes nationales"! Le théâtre n'est pas en dehors des préoccupations des sociologues ni des psychologues pour ne citer que Duvignaud et Freud.

En bref, il aurait fallu expliciter les significations du mot "théâtre », affiner les questions, établir des contacts personnels avec les questionnés. Dans l'état, les résultats de l'enquête donnent une première approche qui reste à compléter, et ne concernent que les départements de lettres modernes, lettres classiques et langues vivantes. Nous remercions donc tous ceux qui ont répondu et espérons que le compte-rendu ne trahit pas leur pensée. Nous avons dû compléter certaines données et espérons ne pas avoir commis d'erreur. Le dépouillement de l'enquête nous amène à préciser ses limites : il s'agit d'un état des lieux, d'une approche générale et non d'une étude minutieuse qui exigerait des précisions chiffrées.

Le théâtre apparaît à la Faculté sous trois formes: celle de l'enseignement, de la recherche, de l'animation ou mieux d'une formation générale de l'étudiant.

\section{- L'enseignement :}

$4 \quad$ Il se présente sous trois aspects :

1. Le DUMST (Diplôme d'Université aux Métiers du Spectacle et du Théâtre). Enseignement à but professionnel en collaboration avec L'Embarcadère (anciennement 
Centre de Rencontres) sera l'objet d'un article spécial dans le prochain numéro de Coulisses.

2. Un enseignement spécifique à but général, les modules et le certificat théâtre sous la responsabilité de la section de littérature comparée.

3. Les programmes concernant le théâtre interviennent dans tous les enseignements de littérature à tous les niveaux.

\section{- La recherche :}

5 Elle se présente soit comme une partie d'un thème général, soit comme le champ majeur de l'étude. Elle est extrêmement variée.

\section{- L'animation :}

6 Quelques enseignants ont voulu faire une synthèse entre des exigences pédagogiques et une recherche expérimentale, ils ont été amenés à créer des structures originales.

\section{L'enseignement}

7 Le théâtre intervient dès le DEUG, dans le cadre des programmes des modules de littérature, française ou étrangère, ancienne ou moderne. Il est rarement traité comme un art en soi, mais comme une manifestation de l'histoire littéraire, ou comme la production d'un auteur. Il peut également intervenir sous forme d'exercices de traduction pour l'apprentissage des langues, ou comme exemples en linguistique.

Le cas du module de littérature comparée, DA 125, est un peu à part. Depuis 1990 il comporte une question de théâtre. En effet, il faisait partie de la formation théorique des étudiants du DUMST. Depuis la rentrée 1994, cette dernière leur est devenue spécifique. L'obligation d'un programme théâtre est donc levée. Il n'en reste pas moins vrai que l'étude de cet art doit demeurer sous une forme spécifique dans les programmes de littérature comparée.

9 Les sections tiennent compte du programme de l'agrégation. En anglais, il comporte toujours une œuvre de Shakespeare. Il est donc nécessaire que, dès la première année, les étudiants soient initiés à cette œuvre ou à cette période. En revanche, celui d'espagnol ou d'italien comporte plutôt des œuvres romanesques. La nécessité de faire figurer des auteurs dramatiques au programme est donc moins prégnante et dépend donc davantage de la spécialité des enseignants en poste.

Les programmes durent, selon les niveaux, les sections et les enseignants d'une à quatre années. Ils sont souvent liés à celui de l'agrégation, particulièrement pour la licence.

11 L'enseignement repose pour l'essentiel sur l'étude de texte, parfois le visionnement de la vidéo du spectacle ou d'une adaptation filmique ; il ne fait pas appel à un intervenant extérieur. Certains d'ailleurs le déplorent, d'autres n'en ressentent pas le besoin, enfin d'autres soulignent le manque d'argent pour le rétribuer.

Les formations ne comportent en général pas de stage pratique exception faite pour F.L.E. où il y a 75 heures de stage obligatoire dans un milieu professionnel, et le module 
Approches des métiers de la culture et de l'information en CLM. Certains étudiants choisissent une structure théâtrale.

Un enseignant reconnaît la valeur d'une telle expérience mais déplore le manque de temps. Une autre souligne la nécessité pour les étudiants d'avoir une approche concrète du théâtre et les encourage vivement à acquérir ces connaissances, hors enseignement, en fréquentant les salles, en assistant aux spectacles.

\section{La part du théâtre dans les DEUG}

Dans les modules de DEUG de Lettres Classiques et Modernes, il est présent dans le cadre de l'histoire de la Littérature française du XVII ${ }^{\text {ème }}$, (D.B111-121, Mme A. Mantéro),

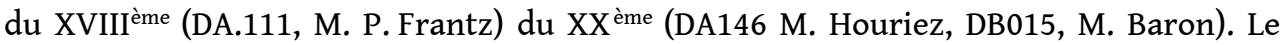
module DA112-114 Langues et littérature grecque et latine (Mme Poulle) propose une lecture cursive d'œuvres dramatiques antiques. En grec, le programme offre une alternance semestrielle : tragédie (M. Fartzoff), comédie (Mme Daude). En latin, Mme Fick lui préfère des études d'extraits sur l'ensemble du cursus. En espagnol dans les modules Littérature et civilisation (DA233, DB234), M. Obrégon traite des œuvres Latino-américaines à raison d'un pièce par semestre. Son homologue anglais, DB224, propose aussi du répertoire anglophone.

\section{La part du théâtre en Licence}

15 Le processus n'apparaît guère différent en licence de Lettres modernes et Classiques. En revanche, les programmes des licences de langues vivantes comportent des œuvres théâtrales. En anglais, dans le C2237 Littérature des pays anglo-saxons, Mme Peltrault traite des œuvres de la Renaissance; en Langue et littérature allemande, M. Jugan, (C2212) du théâtre des XIX ${ }^{\text {ème }}$ et XX ${ }^{\text {ème }}$ siècles; en italien, Mme M. Diaz-Rozzotto, du thêâtre italien au XIX ${ }^{\text {ème }}$ siècle (C2266); en espagnol, (C2242) et en latin (P. Robin) apparaissent des auteurs Valle Inclan et Garcia Lorca pour l'un, Plaute et Sénèque pour l'autre.

\section{Modules et certificat spécifiques :}

\section{L'origine :}

En 1984, la loi Savary proposait aux universités une réforme contractuelle des DEUG avec pour obligation de créer pour tous les étudiants des enseignements obligatoires dont une initiation à une discipline artistique. Dans le cadre des DEUG rénovés la Faculté des Lettres mettait donc en place deux enseignements artistiques : celui du cinéma et celui du théâtre sous la responsabilité de la section de littérature comparée. Le nombre d'étudiants croissant, l'arrivée d'étudiants, titulaires du bac A3 théâtre, ont amené la différenciation des modules et la création d'un certificat.

- Le DEUG :

17 L'originalité vient de ce que l'enseignement pour moitié théorique et pour moitié pratique est assuré avec le concours des professionnels.

- Du texte à la représentation DA147 (C. Tatu et J. Vingler) 
- Histoire du théâtre DA148 (L. Garbagnati et J. Melcore)

- Histoire du théâtre au XX ${ }^{\text {ème }}$ siècle DB148 (L. Garbagnati et J. Melcore)

- Écriture dramatique DB148 (Ph. Baron et J. Melcore).

Si l'approche générale et théorique reste la même, les programmes annuels sont élaborés en fonction de la programmation bisontine et varient chaque année.

\section{- La licence :}

Le certificat optionnel Littérature comparée et théâtre C2131, est uniquement théorique, et assuré par L. Garbagnati et N. Arambasin. Il porte en général sur la notion de genre, cette année la comédie avec Lysistrata, La mégère apprivoisée, Le chapeau de paille d'Italie, La ronde.

\section{Le théâtre et les programmes des concours}

Seuls deux des trente questionnaires reçus ont parlé du CAPES. Tous les autres ont parlé de l'agrégation. La plupart des programmes d'agrégation ne comporte pas de façon régulière une question ou un auteur sur le théâtre. Il faut souligner toutefois la permanence d'une œuvre de Shakespeare dans celle d'anglais et d'une œuvre dramatique en lettres classiques.

\section{La maîtrise :}

\section{Maîtrise à certificat :}

La maitrise F.L.E. comporte un module « Anthropologie culturelle » avec au programme 1994-1995 « Les grands courants esthétiques du XX ${ }^{\text {ème }}$ siècle en France dans le théâtre ». L'enseignement fait appel à une professionnelle : Mme Christine Friedel, dramaturge du Centre Dramatique National.

\section{Les maîtrises à mémoire}

\section{Les certificats de méthodologie :}

Aucun certificat ne porte sur la méthodologie de l'étude du théâtre. Cependant, certains le font intervenir dans leur réflexion.

En anglais le 3223 Méthode de recherche en linguistique anglaise (Mme C. Peltrault), en Allemand le 3201 Méthodologie de la recherche en germanistique (M. G. Jugan), en espagnol (Mme Claire Robin) "le théâtre social», en grec et en latin. Le C2 de littérature comparée (Mme C. Tatu), traite de Iouri Lotman et de la théorie de l'événement.

\section{Les mémoires :}

Les sujets dépendent évidemment des spécialités des enseignants et sont liés à leur recherche personnelle. Ils frappent par leur variété on peut toutefois distinguer des constantes: traduction et analyse, texte et scénographie, théâtre et littérature. Il semble que cette direction soit la plus fréquente. En espagnol sous la direction d'O. Obrégon, les étudiants traduisent et analysent des pièces du répertoire latinoaméricain; en allemand G. Jugan s'intéresse davantage à l'approche d'une pièce par le 
biais de la réception scénique; en grec $\mathrm{M}$. Fartzof aux Tragiques. En littérature comparée, C. Tatu propose des sujets sur le mélodrame et la révolution française, l'articulation entre le XVIII ${ }^{2}$ è et le XIX ${ }^{\text {ème }}$, L. Garbagnati sur la relation entre théâtre et société. En linguistique, Andrée Chauvin dirige un mémoire sur la La poche Parmentier de Pérec ; en littérature française, Mme Miguet sur les deux pièces de Céline, M. Houriez sur Beckett et Ionesco, Mme Mayaux sur l'intertextualité dans le théâtre de Giraudoux.

\section{Au-delà de l'enseignement}

Il n'est pas inutile de signaler qu'au-delà du cursus universitaire, il existe une formation MAFPEN et un cycle "Le regard du spectateur », organisé par le Théâtre Universitaire de Franche- comté, dans le cadre de l'Université ouverte.

\section{La formation MAFPEN}

Elle est organisée par Florent Montaclair. Elle concerne les enseignants du $1^{\mathrm{er}}$ cycle, et étudie "la particularité de l'étude d'une pièce de théâtre au collège à partir de Molière. »

\section{Le cycle Le regard du spectateur}

Comme tous les enseignements dispensés dans le cadre de l'Université Ouverte, il a pour objectif le développement de la culture personnelle sans souci de validation universitaire. Il propose une comparaison, en fonction de la programmation des salles bisontines, entre le texte, sa représentation et le discours du metteur en scène. En 1994, cette confrontation s'est muée en réflexion sur les éléments de la critique.

\section{La recherche :}

\section{La recherche des enseignants}

Recherche solitaire ou collective :

Ces recherches peuvent se dérouler dans le cadre institutionnel d'un centre de recherche, d'un séminaire, ou de manière plus ou moins solitaire.

Les centres de recherche cités sont le Centre de recherche en histoire et littérature des pays de langues européennes, le Centre Jacques Petit, le centre ALSO, le Centre de Recherches Ibériques et Ibéro-américaines, le travail autour d'une publication commune "Le théâtre des romanciers ». Certains enseignants chercheurs sont liés à des centres de recherche d'autres universités par exemple: Paris III pour le théatre latino américain, Aix-en-Provence pour les études Shakespeariennes.

\section{Le théâtre comme partie d'un ensemble :}

La recherche se présente sous deux aspects. Pour certains enseignants-chercheurs, le théâtre intervient comme une partie d'une question plus générale, esthétique, linguistique, poétique générale, étude de réception, pratiques culturelles; pour d'autres comme sujet principal d'une recherche récente ou déjà ancienne. 
Pour Nella Arambasin, le théâtre est un des éléments d'une réflexion générale sur les arts plastiques à la fin du siècle dernier et au début du nôtre. Cécile Daude s'intéresse à la sémiotique littéraire et à celle de l'espace, Philippe Robin à la linguistique et à la métrique des auteurs latins. Marita Gilli se préoccupe de la réception de la révolution française en Allemagne, Gérard Brey des pratiques culturelles en Espagne au XIX ${ }^{\text {ème }}$ et $\mathrm{XX}^{\mathrm{ème}}$ siècle et des auteurs anarchistes espagnols.

Des recherches se mettent en place à l'occasion d'un colloque par exemple celui sur la mythologie de la rébellion ${ }^{1}$ organisé par le Centre d'études des mentalités, ou d'une réflexion commune sur Le théâtre des romanciers ${ }^{2}$.

\section{Le théâtre au centre des recherches}

29 Les recherches permanentes sur le théâtre portent sur le répertoire. P. Frantz étudie celui du XVII ${ }^{\text {ème }}$ au XIX ${ }^{\text {ème }}$ en France, P. Baron celui de la fin du XIX ${ }^{\text {ème }}$ et son interprétation. Elles portent également sur la traduction, l'analyse, la diffusion du répertoire de l'Amérique latine pour Oswlado Obregon ou du Brésil pour Mme Koelher. Claude Peltrault approfondit la réflexion thématique sur la transgression et la subversion dans l'œuvre de Shakespeare et le théâtre de la Renaissance. Gérard Jugan s'intéresse à la réception d'une pièce et d'une mise en scène par la critique. Florent Montaclair veut établir «la spécificité théâtrale dans l'œuvre de Jules Verne » et ce qu'elle comporte de modernité. Michel Fartzoff s'interroge sur la liaison entre les mentalités et les représentations sociales et politiques chez les tragiques grecs et plus particulièrement Eschyle. Marie Miguet travaille sur la permanence et la réécriture des mythes dans les œuvres dramatiques contemporaines.

\section{Les manifestations de la recherche}

\section{Les colloques}

Le résultat d'une recherche s'exprime lors des colloques qui permettent la confrontation. De nombreuses contributions sur le théâtre ont été données dans le cadre de ceux organisées par le Centre de recherche sur les mentalités européennes ${ }^{3}$ ou le centre Jacques Petit ${ }^{4}$. Mais depuis Théâtre et révolution en $1989^{5}$, il n'y en a eu aucun spécialisé sur le théâtre. Il faut donc saluer tout spécialement celui en l'honneur de Dicenta.

\section{Les publications}

L'ouvrage spécifique reste le cas le plus rare. Signalons l'édition critique du Repos du $7^{\text {ème }}$ jour de Paul Claudel par Jacques Houriez et L'Anthologie du théâtre latino-américain d'O. Obregon en collaboration avec Demaingy à paraître en 1996.

Très peu de communications sont publiées dans les Actes de colloques spécifiques au théâtre mais plutôt dans ceux portant sur des questions plus générales. Il en est de même des articles publiées dans les revues de spécialistes ou d'université.

Ces remarques correspondent d'ailleurs à l'analyse des centres d'intérêt des enseignants-chercheurs pour lesquels le théâtre est davantage étudié comme une partie d'un ensemble que pour lui-même. En revanche ceux dont c'est le sujet principal 
ont signé fréquemment des articles dans Théâtre public, La revue d'Histoire du théâtre, et... Coulisses ${ }^{6}$.

\section{Au carrefour de l'enseignement, de la pédagogie et d'une recherche pratique}

Certains enseignants ont été amenés à créer des ateliers de pratique théâtrale indépendante du cursus universitaire, puisque leur fréquentation n'est pas validée. Ainsi, Jutta Jugan a créé avec le concours d'une professionnelle Isabelle Diligent, un atelier hebdomadaire de langue allemande ; Andrew Rossiter relayé par Lee Garner, un théâtre de langue anglaise: le BELT (Besançon English Language Theatre); Lucile Garbagnati, en collaboration avec Joseph Melcore metteur en scène, le Théâtre Universitaire de Franche Comté. Les motivations et de ces activités théâtrales, quelles qu'en soit la diversité, relèvent d'une même considération: la Faculté n'est pas seulement un lieu d'apprentissage intellectuel mais aussi un lieu de formation personnelle.

Ainsi pour Jutta Jugan, l'apprentissage d'une langue passe par sa mise en bouche, un rapport physique et affectif avec le rythme et les sonorités de la langue. Seul le théâtre permet cette mise en situation et mise en condition. Pour Andrew Rossiter, créateur du BELT, il s'agit d'introduire en France une tradition culturelle des universités anglaises, la production d'une pièce. Au-delà du rapport avec la langue, c'est un rapport avec une forme culturelle qui s'établit. Elle permet aussi aux anglophones de mieux s'intégrer. Le T.U.F.C., lui, n'a pas de vocation linguistique mais cherche plutôt à développer la créativité individuelle et la réflexion sur le processus de la production dramatique.

\section{Quels rapports entretenez-vous avec le théâtre?}

La généralité de la question permettait de ne pas répondre (2 questionnaires) ou de poser un point d'interrogation ( 1 questionnaire). Les réponses sont toutefois très éclairantes.

La question a été comprise par les uns : allez-vous souvent au théatre? Bien que les réponses ne soient pas quantifiables, mais on peut en citer une qui les résume : « je suis passionné par le théâtre, mais mon travail m'empêche d'y aller autant que je le voudrais ».

Pour d'autres, il s'agit du rapport entre le spectateur et l'analyse: «Rapport de consommation gourmande... » Mais ils sont plus difficiles lorsqu'il s'agit de travailler une pièce comme texte... et aussi « De velléité de pratique... »

Pour d'autres encore, il s'agit d'un rapport essentiellement professionnel d'enseignant et de chercheur où l'art et le plaisir de la représentation n'interviennent que fort peu. À l'extrême, il y a « la passion, le dégoût total et parfois, hélas, l'indulgence critique !» On ne peut que conclure en citant "spectateur, enseignant, chercheur, vulgarisateur " en complétant par «la nécessité de voir les choses dans le concret en pratiquant le théâtre ». 


\section{Conclusion}

Quelle conclusion donner à ce panorama incomplet et non chiffré, sans comparaison avec d'autres universités, ni avec un modèle idéal. En l'état il donne quelques indications d'ensemble sur l'enseignement et la recherche sur le théâtre à l'Université de Franche-Comté.

L'un et l'autre sont orientés vers le répertoire et la théorie plutôt que vers la pratique et la représentation à l'exception des modules spécifiquement théâtraux. Dans ces conditions ne serait-il pas intéressant d'encourager les étudiants à une ouverture sur la représentation en leur recommandant d'assister aux spectacles et de profiter des avantages du passeport culturel ${ }^{7}$ ?

Les recherches apparaissent comme isolées dans un ensemble. A son niveau, Coulisses pourrait publier les informations qu'on voudrait bien lui communiquer sur les travaux en cours. Ne serait-il pas possible que tous ceux intéressés se retrouvent et se confrontent au sein d'un séminaire ou de journées d'études, qui dans un premier temps pourraient être informelles. Mais qui les organisera?

Certains collègues travaillent sur le théâtre étranger, il y aurait sans doute une dimension à approfondir, dans le cadre des échanges internationaux et plus particulièrement dans le cadre des échanges Erasmus, comme c'est le cas pour le projet avec l'Université de Birmingham.

L'enseignement et la recherche sur le théâtre, à la Faculté des Lettres de Besançon relève d'une question plus générale celle des rapports entre les pratiques et les structures artistiques, voire l'art, et l'Université ou plus généralement l'éducation, question longuement débattue dans d'autres lieux ${ }^{8}$. Elle pose aussi la question du rapport entre savoir et culture. Peter Brook semble résumer la question :

L'art n'est pas nécessairement bénéfique en soi. Un grand chef-d'œuvre présenté d'une certaine manière, peut nous endormir. Présenté d'une autre façon, il peut être une révélation. L'art n'est utile à l'homme et à la société que s'il contient en lui une incitation à l'action; et l'action est un réseau qui s'étend du pain et de la politique à l'être même. ${ }^{9}$

\section{NOTES}

1. Voir dans la rubrique Annonces le descriptif du Colloque Mythologie de la rébellion dans le théâtre en Espagne, en Amérique latine et en Europe (1890-1910)

2. Voir dans la rubrique Publications à paraître

3. Voir les Actes

4. Actes

5. Actes

6. P. Baron, J-P. Colin, V. Grosjean, M.Gilli, G.G.- Holtzer, J. Houriez, G. Jugan, P. Louis, Y. Marin, F. Migeot, C. Peltrault ont déjà publié dans la revue Coulisses.

7. Voir dans la rubrique Vie culturelle: Le passeport culturel

8. Théâtre, éducation et société, in Cahiers théâtre/éducation nº 3 Actes sud-Papiers.

9. Points de suspension, p. 330, Seuil, 1992 